\title{
Phytophthora infestans effector Pi14054 is a novel candidate suppressor of host silencing mechanisms
}

\author{
Ramesh R. Vetukuri • Stephen C. Whisson • \\ Laura J. Grenville-Briggs
}

Accepted: 26 March 2017 / Published online: 10 April 2017

(C) The Author(s) 2017. This article is published with open access at Springerlink.com

\begin{abstract}
Phytophthora infestans is the oomycete pathogen responsible for the devastating late blight disease on potato and tomato. It can also infect the model solanaceous plant Nicotiana benthamiana. P. infestans secretes, and translocates into host cells, an array of RxLR class effectors potentially involved in diverse functions that facilitate infection of host plants. Intensive research efforts are currently focused on determining the role(s) of effectors in promoting late blight disease development, with many experiments carried out in the model plant $N$. benthamiana. In this study we demonstrate that candidate effector Pi14054 has activity as a suppressor of RNA silencing in $N$. benthamiana using an established reporter assay based on the Turnip crinkle virus - green fluorescent protein (TCV-sGFP) system. Pi14054 was able to complement TCV deficient for a viral suppressor of RNA silencing. This is a novel way to identify RNA silencing suppressors that potentially manipulate host immune responses. Transcripts encoding Pi14054 were most abundant during the first $36 \mathrm{~h}$ of infection development, suggesting that expression of this gene is specifically induced
\end{abstract}

Electronic supplementary material The online version of this article (doi:10.1007/s10658-017-1222-9) contains supplementary material, which is available to authorized users.

R. R. Vetukuri $(\bowtie) \cdot$ L. J. Grenville-Briggs

Department of Plant Protection Biology, Swedish University of Agricultural Sciences, Sundsvägen 14, 23053 Alnarp, Sweden e-mail: Ramesh.Vetukuri@slu.se

S. C. Whisson

Cell and Molecular Sciences, The James Hutton Institute, Invergowrie, Dundee DD2 5DA, UK in contact with host tissue to modulate plant defences, as seen with other known effectors. Pi14054 is present in a diverse range of modern aggressive and hyper-aggressive European $P$. infestans isolates, suggesting a potential role for this effector in pathogenicity.

Keywords Suppressors of silencing · Effectors ·

P. infestans $\cdot$ Pi14054

Oomycetes, also known as water molds, are eukaryotic microbes that resemble fungi in their mode of growth and the ecological niches that they occupy. Many members of this group are economically damaging plant pathogens causing serious agricultural or environmental diseases across the world. Phytophthora infestans is the causal agent of late blight on potato and tomato and has significant potential to affect global food security. It can also infect the model plant Nicotiana benthamiana, an experimental host widely used to study disease mechanisms. During interaction with its hosts, $P$. infestans secretes a diverse array of effector proteins that may act either outside host cells (apoplastic effectors) or inside host cells (cytoplasmic effectors) to manipulate host cell structure or functions, to facilitate infection and/or trigger defense responses (reviewed in Schornack et al. 2009). In silico analysis has predicted large classes of effectors within the oomycetes and specifically within $P$. infestans. The RxLR and Crinkle and Necrosis (CRN; crinkler) effector families are characterized by conserved peptide motifs required for translocation into the host (Whisson et al. 2007; Schornack et al. 2010). The large effector repertoire 
of $P$. infestans provides the pathogen with the potential for effector functional redundancy, enabling adaptation to changes in the host environment (such as disease resistance), leading to host resistance breakdown in crop plants, or increased aggressiveness (Fry 2008; Cooke et al. 2012; Vleeshouwers et al. 2011).

Although $P$. infestans is known to manipulate host immune responses by secreting numerous effectors, the functions of relatively few of these effectors have been elucidated so far and the functions of the vast majority of $P$. infestans effectors remain unknown (reviewed in Whisson et al. 2016). In the related soybean pathogen Phytophthora sojae two members of the RxLR class of effectors, PSR1, PSR2 (PsAvh18 and PsAvh146) were recently discovered to act as suppressors of host RNA silencing and promote Phytophthora infection (Qiao et al. 2013). A homolog of PSR2 has been identified in P. infestans (PITG_15152) and several other Phytophthora species (Xiong et al. 2014; de Vries et al. 2017).

RNA silencing is a well-conserved regulatory mechanism in eukaryotes contributing to genome stability, chromatin organization, and disease resistance. It is characterized by non-coding small RNA molecules (sRNA) of 19-40 nt (Holoch and Moazed 2015; Åsman et al. 2016; Vetukuri et al. 2012; Vetukuri et al. 2013). RNA silencing operates both at the transcriptional and post-transcriptional levels. As a counter defense to RNA silencing in host plants, bacteria, viruses and oomycetes employ suppressor proteins that disrupt their host's RNA silencing machinery (Huang et al. 2016). Suppressors of host RNA silencing in oomycetes are known to suppress RNA silencing by inhibiting the biogenesis of small RNAs in general. More specifically, PSR1 was shown to down regulate the accumulation of microRNAs and small interfering RNAs in Arabidopsis, via binding of a host RNA helicase, whilst PSR2 specifically decreases the accumulation of host siRNAs (Qiao et al. 2013; Qiao et al. 2015). The overexpression of both of these $P$. sojae effectors as well as PITG_15152 from $P$. infestans enhances virulence of both these oomycetes and of viruses (Qiao et al. 2013; Xiong et al. 2014). Thus the secretion of silencing suppressors is likely to be a general counter defense mechanism employed by oomycete pathogens.

With the aim of identifying additional suppressors of silencing in $P$. infestans, we have tested a number of putative effectors in our collection (Table S1) using a reporter assay based on the Turnip crinkle virus - green fluorescent protein (TCV-sGFP) system (Powers et al. 2008a, b; Lukhovitskaya et al. 2014). This is a newly established, novel way to identify RNA silencing suppressors that are not detected by other assays either due to the mode of action of the suppressor, or suppression strength (Powers et al. 2008a, b; Lukhovitskaya et al. 2013). The GFP co-infiltration assay (Takeda et al. 2005) is a conventional method used to identify suppressors of silencing. However the GFP co-infiltration assay can only identify suppressors that act at the intracellular level; intercellular suppressors will not be detected (Powers et al. 2008a, b). The TCV-sGFP complementation assay is a robust method based on viral RNA replication compared to the nuclearbased, non-replicating GFP co-infiltration assay. TCV movement from cell-to-cell in $N$. benthamiana requires expression of the capsid protein (CP), a well-known silencing suppressor, which has been extensively characterised. TCV CP (also called p38) has dual functions one, in blocking loading of two argonaute proteins (AGO1 and AGO2); these proteins are pivotal members of RISC complex (RNA-induced silencing complex) aiding in targeting of transcripts destined for degradation. Secondly, TCV CP also binds long dsRNAs to block their processing into the sRNAs required for target degradation (Iki et al. 2017). Thus, TCV CP prevents the degradation of viral transcripts by host silencing machinery. For the TCV-sGFP complementation assay, s wapping the TCV CP gene with a reporter gene for a synthetic GFP (sGFP) blocks the movement of TCV, restricting the virus to single cells or foci consisting of two or three cells (visualized by fluorescence) due to induction of antiviral silencing (Powers et al. 2008a). Complementation with potential suppressors of silencing in trans by Agrobacterium tumefaciens infiltration enables movement of TCV-sGFP and increases the foci (fluorescence) diameter. Thus the ability of a candidate protein to act as a silencing suppressor is confirmed by an increase in fluorescent foci. This method has been used to successfully identify and validate the function of new viral proteins that act as suppressors of host silencing (Powers et al. 2008b; Lukhovitskaya et al. 2013). The present study represents the first report, to our knowledge, of the TCV-sGFP assay to functionally characterize an oomycete silencing suppressor.

All RxLR effectors tested were cloned into Gateway cloning vectors as described in Munch et al. (2015). The primers used for cloning $\mathrm{Pi} 14054$ were (FW 5'CACCATGCGCTGCAATCACACC3' Rv 5'CTAAAAGTCGATTATCGGCTTTTTAC3'). We tested putative RxLR effectors from our collection, (listed in Table S1) along with controls, by syringe infiltrating $N$. benthamiana leaves with A. tumefaciens 
delivering genes encoding candidate silencing suppressors under control of the $35 \mathrm{~S}$ promotor from Cauliflower Mosaic Virus (CaMV) (Lukhovitskaya et al. 2014). After $24 \mathrm{~h}$, the pre-infiltrated leaves were mechanically rub-inoculated with TCV-sGFP infectious RNA transcripts (Powers et al. 2008a, b). Foci were viewed by fluorescence microscopy at 3 days post inoculation (dpi). A. tumefaciens strains carrying an empty plasmid (EP) were used as a negative control. A plasmid expressing HcPro, a well-known strong silencing suppressor from potato virus A (PVA), was used as a positive control (Savenkov and Valkonen 2001). We monitored the movement of TCV-sGFP and found that the fluorescent foci of HcPro and Pi14054 penetrated up to 20 cells (Fig. 1a-c) compared to the negative control (empty plasmid), which was restricted to single cells. No other effector candidates exhibited any increase in fluorescence foci. This demonstrates that Pi14054 has activity as a suppressor of RNA silencing, enabling the movement of TCV-sGFP with increase in the foci (fluorescence) diameter. The experiment was repeated on four independent occasions and yielded consistent results on all four occasions. Previously discovered suppressors of silencing in oomycetes are known to suppress RNA silencing in plants by inhibiting biogenesis of small RNAs (Qiao et al. 2013). It is possible that Pi14054 acts in a similar manner, however the exact mechanism of suppression activity of Pi14054 is yet to be determined.

P. infestans RxLR effectors typically exhibit marked transcript accumulation during pathogenesis (Whisson et al. 2007; Haas et al. 2009). To investigate if Pi14054
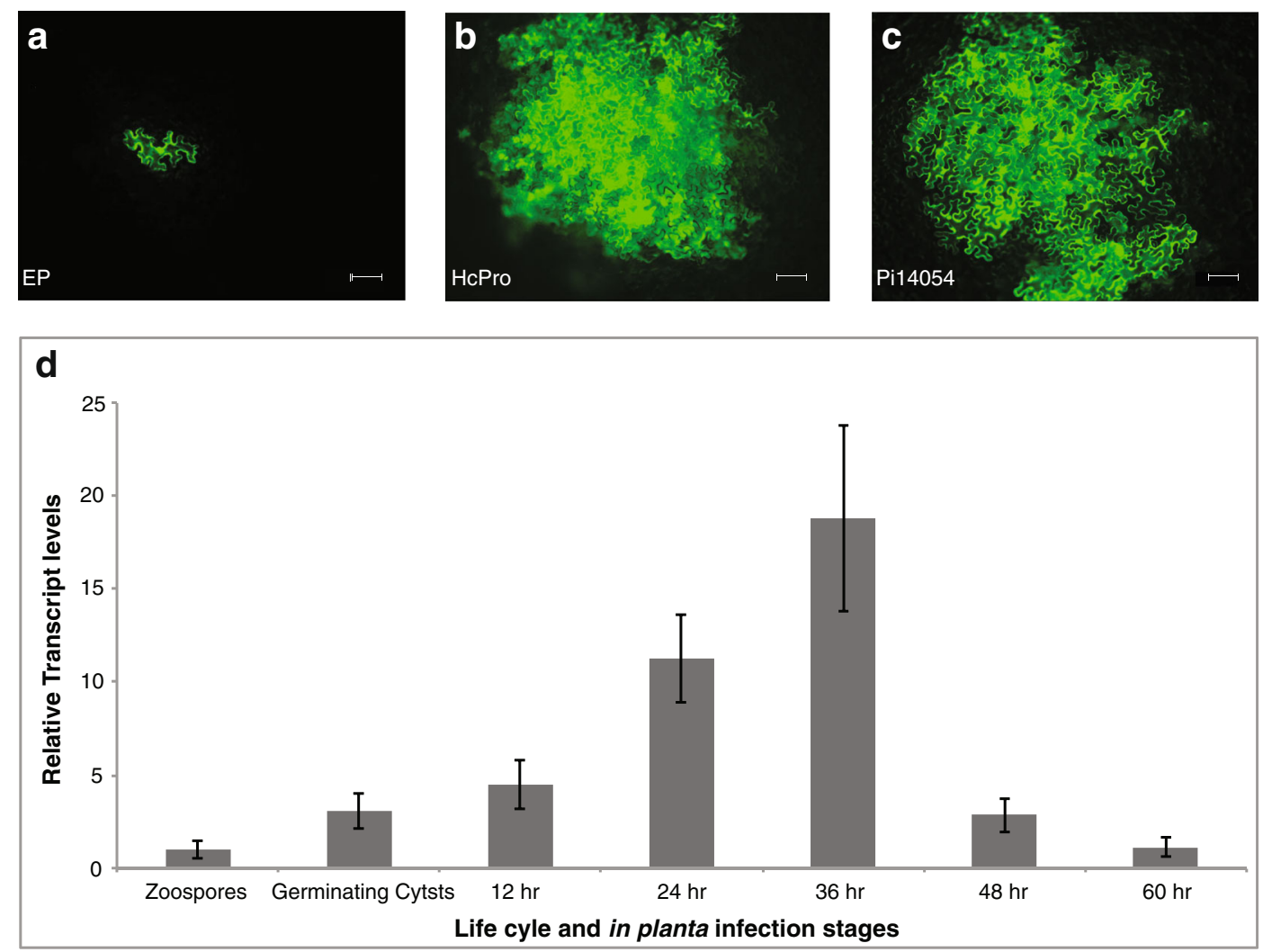

Fig. 1 a,b,c Phytophthora infestans effector Pi14054 suppresses host silencing in $N$. benthamiana. $(\mathrm{a}, \mathrm{b}, \mathrm{c})$ Fluorescence microscopy imaging of the TCV-sGFP complementation assay with constructs expressing empty plasmid (EP), HcPro and Pi14054 as indicated. Preinfiltrated Agrobacterium carrying EP (a) did not complement the movement of TCV-sGFP out of the single cell, whilst pre-infiltrated HcPro (b) and Pi14054 (c) gene constructs complemented the movement of TCV-sGFP to neighbouring cells. Images were taken at 3 days post-inoculation (dpi). Bars $=100 \mu \mathrm{m}$. (d) Relative transcript abundance (qRT-PCR) of effector Pi14054 at different life cycle stages and infection time points in $P$. infestans-potato infection. The transcript profiles are shown in pre-infection stages (zoospores and germinating cysts) and at 12, 24, 36, 48, and $60 \mathrm{~h}$ post-inoculation (hpi) of potato cultivar Bintje relative to the mRNA level of Actin A and Ubiquitin in the zoospore pre-infection stage. All calculations and statistical analyses were carried out as described in Vetukuri et al. (2012). Error bars represent confidence intervals calculated from three biological replicates 
transcripts accumulated during $P$. infestans infection of potato leaves, quantitative real-time reverse transcription polymerase chain reaction (qRT-PCR) was carried out. Susceptible potato cultivar Bintje was used for $P$. infestans (isolate 88069) infection assays with zoospores as the inoculum source. Infection assays, preparation of isolate 88069 life cycle stages, and sample collection were carried out as described (Vetukuri et al. 2011a). Total RNA preparations, DNase treatment, cDNA synthesis, qRT-PCR assay, and calculations were performed as described previously (Vetukuri et al. 2012). qRT-PCR assays were performed with Actin A (PITG_15117) (Vetukuri et al. 2012) and Ubiquitin (PITG_07230) (Jahan et al. 2015) as endogenous controls. Primers were designed and optimized to amplify Pi14054 (Fw 5' GCGAAATGGGGAACTACGAC 3' and Rv 5' TGAGCCTTTAGAGCTTCCCC 3') and transcript levels were analyzed as described previously (Vetukuri et al. 2012). Transcript abundance in germinating cysts and infection time points on Bintje (12, 24, 36,48 and $60 \mathrm{~h}$ post inoculation (hpi)) was compared with the level of transcript in the calibrator stage (zoospores of isolate 88069). The calibrator samples were assigned the relative value of 1.0 , to allow comparisons to be made between different infection time points and life cycle stages. All qRT-PCR amplifications were repeated on independent occasions with cDNA generated from three independent biological replicates. The transcript accumulation of Pi14054 in infection time points increased several fold compared to levels in the zoospore stage (Fig. 1d). This corresponds to a five-fold increase at $12 \mathrm{hpi}$, a ten-fold increase at $24 \mathrm{hpi}$, and a peak of a 20 -fold increase at 36 hpi. Transcript levels of Pi14054 were lower by 48 hpi. This suggests that Pi14054 functions early in the infection process while the interaction is still predominantly biotrophic.

Pi14054 is annotated in NCBI GenBank as a secreted RxLR effector. It possesses a predicted signal peptide for secretion (Signal P 4.0; http://www.cbs.dtu. $\mathrm{dk} /$ services/SignalP/) (Fig. 2a) and a predicted bipartite nuclear localization signal (cNLS Mapper; http://nlsmapper.iab.keio.ac.jp/cgi-bin/NLS_Mapper_form.cgi). However, despite being annotated as an RxLR effector, it does not possess an intact canonical RxLR motif (PLVR instead), considered to be involved in the translocation of the peptide into host plant cells. It does possess an EER motif close to the predicted signal peptide cleavage site. A similar organisation is also
Fig. 2 a Diagrammatic representation of protein domains in Pi14054. Signal peptide region, EER motif, and predicted bipartite nuclear localization signal (NLS) are shown. Numbers above the diagram indicate amino acid positions. (b) Protein sequence alignment with possible orthologs of Phytophthora infestans effector Pi14054. Individual signal peptide regions in each of the three sequences are shown in green. Highlighted in a blue box is the missing/ mutated RxLR motif from Pi14054, compared to PPTG_09764 from P. parasitica and PHYCI 91937 from $P$. cinnamomi. EER motifs are also shown in blue. Predicted bipartite NLS for Pi14054 (185-216 amino acids), PPTG_09764 (205-234) and PPTG_09764 (218-244) are indicated in black. Alignments were produced using the CLC workbench software multiple protein alignment tool. (c) PCR analyses of Pi14054 in different $P$. infestans isolates showing presence of Pi14054 and Actin A in the genomes of all isolates tested. Actin A was used as PCR positive control and no template control (NTC) as negative control. Amplicon sizes of Pi14054 and Actin A are 690 and 209 (bp), respectively, are shown at right

found in the ATR5 effector from Hyaloperonospora arabidopsidis, recognised by the cytoplasmic resistance protein RPP5 from Arabidopsis thaliana (Bailey et al. 2011). Using BLASTP and default search parameters, possible orthologs of Pi14054 (Fig. 2b) can be found in P. parasitica (RxLR effector PPTG_09764-t26_1-p1; 71\% identity) and P. cinnamomi (PHYCI_91937T0-p1; 53\% identity). Other less similar sequences were found in $P$. ramorum, P. parasitica, P. capsici, and P. sojae (26-41\% identity). No significant sequence similarity was found between Pi14054 and previously described P. sojae silencing suppressors PsPSR1, PsPSR2 or to P. infestans homo$\log$ of PsPSR2, PITG_15152. Outside the Phytophthora genus, there were no other sequences found with significant similarity to Pi14054.

Having established that Pi14054 displayed RNA silencing suppression activity in planta, we next tested for the presence/absence of this gene in a diverse collection of European P. infestans isolates. This collection contains isolates from the UK, the Netherlands and Sweden including aggressive and hyper-aggressive isolates. P. infestans isolates were cultured in liquid pea medium and mycelium harvested as described previously (Vetukuri et al. 2012). DNA extraction from frozen mycelium was carried out as reported previously (Löbmann et al. 2016; Kushwaha et al. 2017). PCR reactions were performed with Pi14054 primers (Fw 5' CACCATGCGCTGCAATCACACC3', Rv 5 ' CTAAAAGTCGATTATCGGCTTTTTAC 3), and Actin A primers (Vetukuri et al. 2011b) to detect Pi14054 and Actin A (PCR control) respectively. PCR conditions were as described in Vetukuri et al. (2011b). 
a

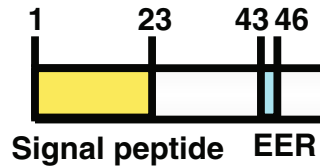

185

216229

Signal peptide

b PITG 14054 MRCNHTLCVV AITTFLVSWSO TLSTPVESRR TE- SP. . . I

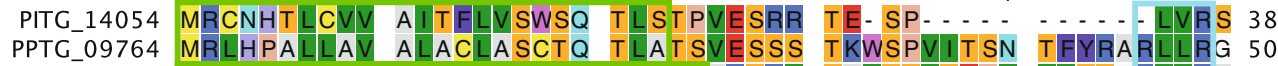
PHYCI_91937 MRIFYATMMAT A-AFLAG-GE SLAAGAKSRV SAVTSITDPI VVSSGRLLRA 48 Consensus MRXXXTLXAV AXAFLASXXQ TLATXVESRX TXXSPXXXXX XXXXXRLLRX

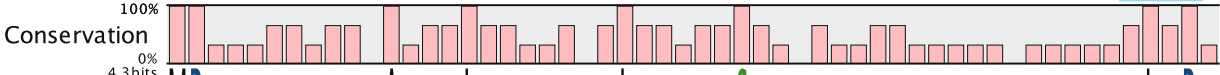

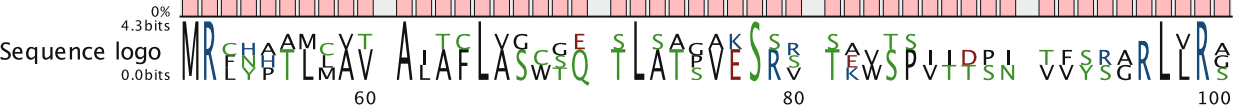
60

PITC_14054 VSA-T- - - EE RNIFSQTAEA VAKWGTTTAL LNLGKTDDEV KKILGLEK- L 83 PPTC_09764 VAT-SDDA EE RDFF SQMAEA VAKWGTTTAL LSLGKTDDEA KKILGLEK- L 98 PHYCI_91937 AKSYSTDTEE RGFISQFVDD VTKMGSVTFW VNTGKTDDYV KDVLHIDKTL 98

Consensus VXX-SXDXEE RXFFSQXAEA VAKWGTTTAL LNLGKTDDEV KK I LGLEK-L Conservation

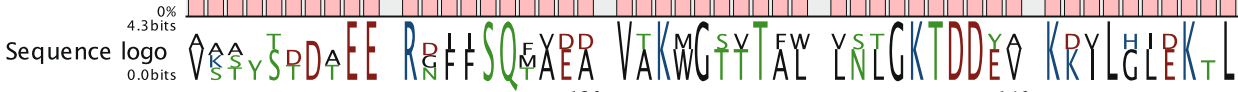
120 140 PITG_14054 SGEALKAHSN YHLLDDFITK LRDRKVTGWL HKDTTTDEVW KTLQLDDLFA 133 PPTC_09764 SGEALKSHAN YHILDEFLSK LRSRKVTQWL NKDTTTDEVW KALQLDDLST 148 PHYCI_91937 TGAALKAHPN YKIFEEFFEK MDARKVDAWI NKDTPTAEVW TILGLD- - - 144

Consensus SGEALKAHXN YHI LDEFXXK LRXRKVTXWL NKDTTTDEVW KXLQLDDLXX Conservation

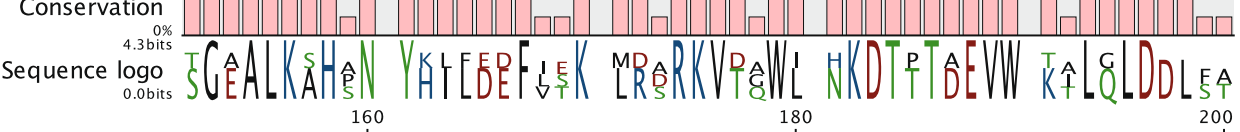
200 PITG_14054 KLDAKEFRHS DELKTYVQYV KKLDDDIWNY KRASFEPDSS SPLELAVKIH 183 PPTC_09764 KLDAKEFKQS EALKTYVEYYV KKLDDDIVKF KRASFEPDNS SPLELAVKIH 198 PHYCI_91937 KLTKQELKES DALTTYIRYA TKLDDEIWHY KRASFEPEIS SATELSVKVR 194

Consensus KLDAKEFKXS DALKTYVXYV KKLDDDIWXY KRASFEPDXS SPLELAVKIH

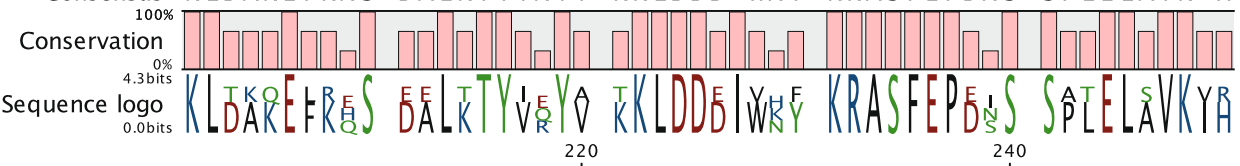

PITG_14054 I WAKAKIRISSW HIVILIEMMGNNA LIKGSKNRKIFIY REIYLILLI IKGK KP I I DF 229 PPTG 09764 IWAKAGRDPA HALIEIIMGMNA LIKGSKNRKIFIY EIEIFLD LIINGR KP IIIDY 244 PHYCI_91937 IWANADRPSW YVILEMMGKNA KHGNPNYIEYYIY QLIFQKILII|KAK KQRILIVN 240

Consensus IWAKAXRPSW HVLEMMGXNA LKGSKNRKFY XEFLXLIKGK KPIIDX

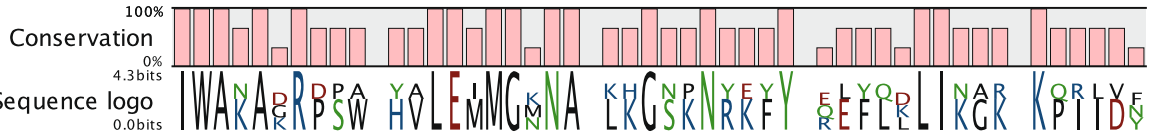

C

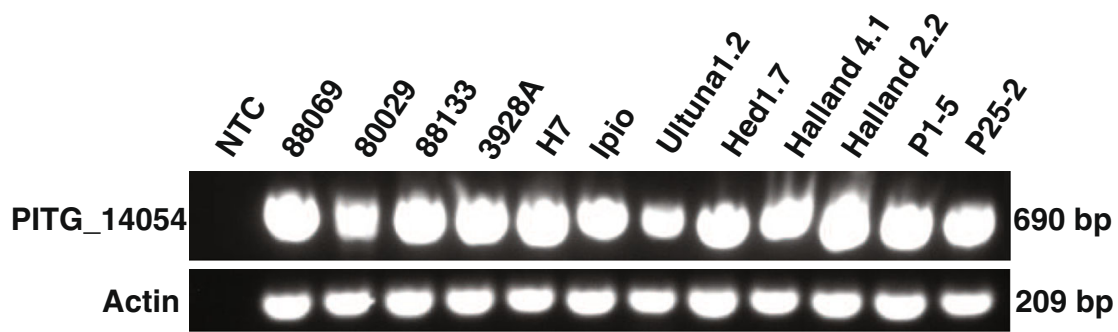


The expected PCR products were 690 bp (Pi14054) and 209 bp (Actin A). Pi14054 was detected in all the European isolates tested (Fig. 2c) suggesting the importance of the effector in silencing suppression activity during pathogenesis.

RxLR class effectors that can act to suppress host plant RNA silencing mechanisms have been described from P. sojae, with PSR2 orthologs being present in a variety of Phytophthora species, including P. infestans (Xiong et al. 2014). Here we have shown that $P$. infestans possesses an additional predicted secreted effector with silencing suppression activity. Plants possess multiple RNA silencing pathways with potential linkage to disease resistance and it appears likely that successful pathogens must suppress or otherwise manipulate these pathways to their advantage (Huang et al. 2016). Further work will focus on identifying the host protein(s) that potentially interact with Pi14054, how it may impact host defence functioning, and its importance to pathogenicity in $P$. infestans. Although there are now at least two $P$. infestans effectors with silencing suppression activity, it is possible that there are more to be discovered within the complex effector repertoire of this important plant pathogen.

Acknowledgements We are grateful to Laura Vetukuri for help in the lab. Swedish Research Council, Formas, the Swedish Foundation for Strategic Research (SSF) and Kungl. Skogs- och Lantbruksakademien, (KSLA) supported this work. SCW was supported by funding from the Scottish Government Rural and Environmental Science and Analytical Services (RESAS). The funders had no role in study design, data collection and analysis, decision to publish, or preparation of the manuscript.

Open Access This article is distributed under the terms of the Creative Commons Attribution 4.0 International License (http:// creativecommons.org/licenses/by/4.0/), which permits unrestricted use, distribution, and reproduction in any medium, provided you give appropriate credit to the original author(s) and the source, provide a link to the Creative Commons license, and indicate if changes were made.

\section{References}

Åsman, A. K. M., Fogelqvist, J., Vetukuri, R. R., \& Dixelius, C. (2016). Phytophthora infestans Argonaute 1 binds microRNA and small RNAs from effector genes and transposable elements. New Phytologist, 211, 993-1007.

Bailey, K., Çevik, V., Holton, N., Byrne-Richardson, J., Sohn, K. H., Coates, M., et al. (2011). Molecular cloning of ATR5Emoy2 from Hyaloperonospora arabidopsidis, an avirulence determinant that triggers RPP5-mediated defense in Arabidopsis. Molecular Plant-Microbe Interactions. doi:10.1094/MPMI-12-10-0278.
Cooke, D. E. L., Cano, L. M., Raffaele, S., Bain, R. A., Cooke, L. R., Etherington, G. J., Deahl, K. L., Farrer, R. A., Gilroy, E. M., Goss, E. M., Grünwald, N. J., Hein, I., MacLean, D., McNicol, J. W., Randall, E., Oliva, R. F., Pel, M. A., Shaw, D. S., Squires, J. N., Taylor, M. C., Vleeshouwers, V. G. A. A., Birch, P. R. J., Lees, A. K., \& Kamoun, S. (2012). Genome analyses of an aggressive and invasive lineage of the Irish potato famine pathogen. PLoS Pathogens, 8, e1002940.

de Vries, S., von Dahlen, J. K., Uhlmann, C., Schnake, A., Kloesges, T., \& Rose, L. E. (2017). Signatures of selection and host-adapted gene expression of the Phytophthora infestans RNA silencing suppressor PSR2. Molecular Plant Pathology, 18, 110-124.

Fry, W. (2008). Phytophthora infestans: The plant (and R gene) destroyer. Molecular Plant Pathology. doi:10.1111/j.13643703.2007.00465.x.

Haas, B. J., Kamoun, S., Zody, M. C., Jiang, R. H., Handsaker, R. E., Cano, L. M., et al. (2009). Genome sequence and analysis of the Irish potato famine pathogen Phytophthora infestans. Nature. doi:10.1038/nature08358.

Holoch, D., \& Moazed, D. (2015). RNA-mediated epigenetic regulation of gene expression. Nature Reviews Genetics. doi:10.1038/nrg3863.

Huang, J., Yang, M., Lu, L., \& Zhang, X. (2016). Diverse functions of small RNAs in different plant-pathogen communications. Frontiers in Microbiology. doi:10.3389 /fmicb.2016.01552.

Iki, T., Tschopp, M.-A., \& Voinnet, O. (2017). Biochemical and genetic functional dissection of the P38 viral suppressor of RNA silencing. RNA. doi:10.1261/rna.060434.116.

Jahan, S. N., Åsman, A. K., Corcoran, P., Fogelqvist, J., Vetukuri, R. R., \& Dixelius, C. (2015). Plant-mediated gene silencing restricts growth of the potato late blight pathogen Phytophthora infestans. Journal of Experimental Botany. doi:10.1093/jxb/erv094.

Kushwaha, S. K., Vetukuri, R. R., \& Grenville-Briggs, L. J. (2017). Draft genome sequence of the mycoparasitic oomycete Pythium periplocum strain CBS 532.74. Genome announcements. doi:10.1125/genomeA.00057-17.

Löbmann, M. T., Vetukuri, R. R., de Zinger, L., Alsanius, B. W., Grenville-Briggs, L. J., \& Walter, A. J. (2016). The occurrence of pathogen suppressive soils in Sweden in relation to soil biota, soil properties, and farming practices. Applied Soil Ecology. doi:10.1016/j.apsoil.2016.05.011.

Lukhovitskaya, N. I., Thaduri, S., Garushyants, S. K., Torrance, L., \& Savenkov, E. I. (2013). Deciphering the mechanism of defective interfering RNA (DI RNA) biogenesis reveals that a viral protein and the DI RNA act antagonistically in virus infection. Journal of Virology, 87, 6091-6103.

Lukhovitskaya, N. I., Vetukuri, R. R., Sama, I., Thaduri, S., Solovyev, A. G., \& Savenkov, E. I. (2014). A viral transcription factor exhibits antiviral RNA silencing suppression activity independent of its nuclear localization. Journal of General Virology. doi:10.1099/vir.0.067884-0.

Munch, D., Teh, O.-K., Malinovsky, F. G., Liu, Q., Vetukuri, R. R., El Kasmi, F., et al. (2015). Retromer contributes to immunity-associated cell death in Arabidopsis. The Plant Cell. doi:10.1105/tpc.114.132043.

Powers, J. G., Sit, T. L., Qu, F., Morris, T. J., Kim, K.-H., \& Lommel, S. A. (2008a). A versatile assay for the 
identification of RNA silencing suppressors based on complementation of viral movement. Molecular Plant-Microbe Interactions. doi: 10.1094/MPMI-21-7-0879 .

Powers, J. G., Sit, T. L., Heinsohn, C., George, C. G., Kim, K.-H., \& Lommel, S. A. (2008b). The red clover necrotic mosaic virus RNA-2 encoded movement protein is a second suppressor of RNA silencing. Virology, 381, 277-286.

Qiao, Y., Liu, L., Xiong, Q., Flores, C., Wong, J., Shi, J., et al. (2013). Oomycete pathogens encode RNA silencing suppressors. Nature Genetics. doi:10.1038/ng.2525.

Qiao, Y., Shi, J., Zhai, Y., Hou, Y., \& Ma, W. (2015). Phytophthora effector targets a novel component of small RNA pathway in plants to promote infection. Proceedings of the National Academy of Sciences, 112, 5850-5855.

Savenkov, E. I., \& Valkonen, J. P. T. (2001). Potyviral helpercomponent proteinase expressed in transgenic plants enhances titers of potato leaf roll virus but does not alleviate its phloem limitation. Virology. doi:10.1006/viro.2000.0838.

Schornack, S., Huitema, E., Cano, L. M., Bozkurt, T. O., Oliva, R., Van Damme, M., et al. (2009). Ten things to know about oomycete effectors. Molecular Plant Pathology. doi:10.1111 /j.1364-3703.2009.00593.x.

Schornack, S., van Damme, M., Bozkurt, T. O., Cano, L. M., Smoker, M., Thines, M., Gaulin, E., Kamoun, S., Huitema, E. (2010). Ancient class of translocated oomycete effectors targets the host nucleus. Proceedings of the National Academy of Sciences 107:17421-17426

Takeda, A., Tsukuda, M., Mizumoto, H., Okamoto, K., Kaido, M., Mise, K., \& Okuno, T. (2005). A plant RNA virus suppresses RNA silencing through viral RNA replication. The EMBO Journal, 24, 3147-3157.

Vetukuri, R. R., Avrova, A. O., Grenville-Briggs, L. J., Van West, P., Söderbom, F., Savenkov, E. I., et al. (2011a). Evidence for involvement of dicer-like Argonaute and histone deacetylase proteins in gene silencing in Phytophthora infestans.
Molecular Plant Pathology. doi:10.1111/j.13643703.2011.00710.x.

Vetukuri, R. R., Tian, Z., Avrova, A. O., Savenkov, E. I., Dixelius, C., \& Whisson, S. C. (2011b). Silencing of the PiAvr3a effector-encoding gene from Phytophthora infestans by transcriptional fusion to a short interspersed element. Fungal Biology. doi:10.1016/j.funbio.2011.08.007.

Vetukuri, R. R., Åsman, A. K. M., Tellgren-Roth, C., Jahan, S. N., Reimegård, J., Fogelqvist, J., et al. (2012). Evidence for small RNAs homologous to effector-encoding genes and transposable elements in the oomycete Phytophthora infestans. PloS One. doi:10.1371/journal.pone.0051399.

Vetukuri, R. R., Åsman, A. K. M., Jahan, S. N., Avrova, A. O., Whisson, S. C., \& Dixelius, C. (2013). Phenotypic diversification by gene silencing in Phytophthora plant pathogens. Communicative \& Integrative Biology. doi:10.4161 /cib.25890.

Vleeshouwers, V. G. A. A., Raffaele, S., Vossen, J. H., Champouret, N., Oliva, R., Segretin, M. E., et al. (2011). Understanding and exploiting late blight resistance in the age of effectors. Annual Review of Phytopathology. doi:10.1146 /annurev-phyto-072910-095326.

Whisson, S. C., Boevink, P. C., Moleleki, L., Avrova, A. O., Morales, J. G., Gilroy, E. M., et al. (2007). A translocation signal for delivery of oomycete effector proteins into host plant cells. Nature. doi:10.1038/nature06203.

Whisson, S. C., Boevink, P. C., Wang, S., \& Birch, P. R. J. (2016). The cell biology of late blight disease. Current Opinion in Microbiology. doi:10.1016/j.mib.2016.09.002.

Xiong, Q., Ye, W., Choi, D., Wong, J., Qiao, Y., Tao, K., et al. (2014). Phytophthora suppressor of RNA silencing 2 is a conserved RxLR effector that promotes infection in soybean and Arabidopsis thaliana. Molecular Plant-Microbe Interactions. doi:10.1094/MPMI-06-14-0190-R. 